\title{
Significados da atenção à saúde do trabalhador com diabetes atribuídos pelos adoecidos e profissionais de saúde ${ }^{1}$
}

Beatriz Cardoso Lobato²

Carla Regina Souza Teixeira³

Márica Maria Faria Zago ${ }^{3}$

Maria Lúcia Zanetti3

Regina Yoneko Dakuzaku Carretta ${ }^{4}$

Carla Silva Santana ${ }^{4}$

doi: 10.11144/Javeriana.ie19-2.sast

Como citar: Lobato B, Teixeira CR, Zago MM, Zanetti ML, Carretta RY, Santana C. Significados da atenção à saúde do trabalhador com diabetes atribuídos pelos adoecidos e profissionais de saúde. Investig Enferm Imagen Desarr. 2017;19(2):177-94. http:// dx.doi.org/10.11144/Javeriana.ie19-2.sast

1. Artigo original de pesquisa. Data de recebido: 30 de abril de 2015. Data de aceitação: 8 de agosto de 2016.

2. Curso de Terapia Ocupacional, Universidade Federal do Triangulo Mineiro, Brasil. Correio eletrônico: beatriz@ics.uftm.edu.br

3. Escola de Enfermagem de Ribeirão Preto, Universidade de São Paulo, Brasil. Correioa eletrônicos: carlarst@eerp.usp.br,mmfzago@eerp.usp.br, zanetti@eerp.usp.br

4. Curso de Terapia Ocupacional, Faculdade de Medicina de Ribeirão Preto, Universidade de São Paulo, Brasil. Correios eletrônicos: reginadc@fmrp.usp.br, carla.santana@fmrp.usp.br 


\section{Resumo}

Objetivo: Compreender os significados atribuídos pelas pessoas com diabetes e profissionais de saúde a atenção à saúde do trabalhador com diabetes. Método: Participaram do estudo 19 pessoas, das quais onze com diabetes e oito profissionais de saúde de um Centro de Saúde Escola do interior de São Paulo, Brasil. Utilizou-se o referencial teórico do interacionismo simbólico e o referencial metodológico da teoria fundamentada nos dados. Resultados: Para os participantes do estudo a atenção à saúde da pessoa com diabetes que trabalha é compreendida como uma atenção fragmentada, centrada em ações biológicas e prescritivas, que não abarcam as implicações emocionais e sociais desta condição crônica e não oferecem suporte para lidar com esta condição no contexto do trabalho. Conclusões: Identifica-se a necessidade de intervenções ampliadas em saúde que considerem as pessoas com diabetes em sua integralidade, além de ações que visam à criação de dispositivos legais capazes de subsidiar a realização de ações de autocuidado no contexto do trabalho.

Palavras-chave: diabetes mellitus; saúde; trabalho; serviços de saúde; pessoal de saúde

\section{Meanings of Health Care to Workers with Diabetes Assigned by Diseased and Health Professionals}

\section{Abstract}

Objective: To understand the meanings attributed by people with diabetes and healthcare professionals attention to the health worker with diabetes. Method: The study included 19 people, of which eleven with diabetes and eight health professionals at a Health Center of São Paulo, Brazil. We used the theoretical framework of symbolic interactionism and the methodological framework of Grounded Theory. Results: For the study, participants attention to health of people with diabetes who works is understood as a fragmented care, focused on biological and prescriptive actions, which do not cover the emotional and social implications of this chronic condition and provide no support to deal with this condition in the work context. Conclusions: Identifies the need for expanded health interventions that consider people with diabetes in their entirety and to create legal devices that support the achievement of self-care actions in the workplace.

Keywords: diabetes mellitus; health; work; health services; health personnel 


\section{Significados de la atención a la salud del trabajador con diabetes asignados por los enfermos y los profesionales de salud}

\section{Resumen}

Objetivo: Comprender los significados asignados por las personas con diabetes y por los profesionales de salud a la atención a la salud del trabajador con diabetes. Método: El estudio tuvo la participación de 19 personas, de las cuales 11 eran pacientes diabéticos y 8 profesionales de la salud en un Centro de Salud Escuela del interior de São Paulo, Brasil. Se utilizó el referencial teórico del interaccionismo simbólico y el referencial metodológico de la teoría fundamentada en los datos. Resultados: Para los participantes de este estudio, la atención a la salud de la persona con diabetes que trabaja es comprendida como una atención fragmentada, enfocada en acciones biológicas y prescriptivas, que no cubren las implicaciones emocionales y sociales de esta condición crónica y no ofrecen apoyo para hacer frente a dicha condición en el contexto de trabajo. Conclusiones: Se identifica la necesidad de intervenciones ampliadas en la salud que consideren a las personas con diabetes en su integralidad, además de acciones dirigidas a crear recursos legales capaces de apoyar la realización de acciones de autoatención en el contexto de trabajo.

Palabras clave: diabetes mellitus; salud; trabajo; servicios de salud; personal de salud 


\section{Introdução}

O diabetes mellitus (DM) é uma condição crônica que tem recebido crescente destaque devido a sua elevada prevalência nas diferentes faixas etárias $(1,2)$, que conduz a um número considerável de pessoas trabalhando e vivendo com esta condição (3).

Estudos (3-7) mostram que o diabetes tem um impacto negativo no trabalho por afetar a produtividade e a renda dos trabalhadores em decorrência de dias perdidos de trabalho, devido a complicações crônicas decorrentes da doença, que podem limitar as habilidades do indivíduo para o trabalho e dificultar sua contratação, tendo como consequência a aposentadoria precoce.

Torna-se importante compreender que o trabalhador, ao receber o diagnóstico de uma condição crônica como o diabetes, precisa desenvolver estratégias para conciliar os cuidados necessários relacionados à manutenção de sua saúde com as exigências relacionadas ao trabalho. O trabalho pode comprometer o autocuidado e o controle do diabetes, ao dificultar o seguimento das restrições alimentares, ao prolongar a jornada de trabalho, ao exigir a realização de esforços físicos, dentre outros fatores. Além disto, a falta de informação dos empregadores a respeito da doença e de seu tratamento favorece a incompreensão destes em relação às necessidades da pessoa com diabetes no ambiente de trabalho (5-8).

A superação das barreiras encontradas no ambiente de trabalho, visando o adequado manejo do DM, pode ocorrer a partir de acomodações necessárias. Para a Associação Americana de Diabetes (ADA) (9) estas acomodações referem-se a pausas no trabalho para administração de insulina ou para realizar os testes dos niveis séricos de glicemia, disponibilidade de local adequado para o armazenamento de insulina, bem como para a sua administração. Em geral as adaptações são simples e de baixo custo.

Nesta perspectiva, a atenção em saúde tem um papel fundamental na orientação da pessoa com DM que trabalha no intuito de auxiliar na identificação e realização das adaptações necessárias em sua rotina de trabalho, de forma a conciliá-la com as ações de autocuidado. Desta forma, objetivouse compreender os significados atribuídos pelos adoecidos e profissionais de saúde a atenção à saúde prestada à pessoa com diabetes que trabalha.

\section{Método}

Trata-se de um estudo qualitativo com referencial teórico no interacionismo simbólico (IS) e referencial metodológico na teoria fundamentada nos dados (TFD). O IS possibilita a compreensão do modo como os indivíduos interpretam os objetos e as outras pessoas com as quais interagem e como tal processo de interpretação conduz o comportamento individual em situações específicas (10-11). Para a compreensão dos significados atribuídos a experiência de ser trabalhador com diabetes utilizou-se como referencial metodológico a TFD que possibilitou ao pesquisador compreender a experiência dos participantes da pesquisa a partir de suas perspectivas (10).

A pesquisa foi realizada em um Centro de Saúde Escola (CSE), localizado em um município do interior de São Paulo, que presta atendimento de nível ambulatorial por uma equipe multiprofissional. Neste serviço é realizado 
também o grupo de educação em diabetes mellitus. O CSE é um serviço de referência no município por reunir profissionais de saúde de diversas áreas e com experiência na atenção à pessoa com diabetes mellitus. Deste modo, o CSE agrega características importantes para o desenvolvimento do estudo.

A coleta de dados iniciou-se com a inserção da pesquisadora no serviço, por meio da participação no grupo de educação em diabetes, que possibilitou a aproximação das pessoas em seguimento pelo serviço, bem como dos profissionais de saúde. O início da coleta de dados ocorreu mediante a aprovação do estudo pelo Comitê de Ética em Pesquisa (Parecer número 1469/2011).

O processo de coleta de dados foi orientado pelas diretrizes da TFD, que se baseia na amostragem teórica, a qual não define previamente o quantitativo de sujeitos que participarão da pesquisa, sendo este determinado pela saturação teórica, na qual o pesquisador procede com a coleta de dados até que ocorra a repetição dos mesmos $(10,12)$.

Participaram do estudo onze pessoas com diabetes mellitus tipo 2 e oito profissionais de saúde, totalizando 19 participantes para que fosse atingida a saturação teórica. Para as pessoas com diabetes, definiu-se como critério de inclusão no estudo pessoas com diagnóstico de diabetes mellitus tipo 2 , em idade produtiva (de 20 a 65 anos) e que apresentavam experiência do diabetes no contexto de trabalho. Para os profissionais de saúde, definiu-se como critério de inclusão ter experiência de intervenção com pessoas com diabetes mellitus no âmbito do serviço público de saúde.

O grupo de pessoas com diabetes foi composto por cinco homens e seis mulheres, com tempo de diagnóstico de DM de um a 14 anos. Todos os entrevistados utilizavam antidiabético oral e apenas dois deles utilizavam também insulina. Quanto ao vínculo de trabalho, sete pessoas apresentavam vínculo formal, em atividades do setor de serviços como: auxiliar de enfermagem, vigia, auxiliar de cobrança e secretária; duas apresentavam vinculo informal, desempenhando atividades como diaristas; e duas eram aposentadas, mas tiveram experiência de vivenciar o diabetes no trabalho em atividades como motorista de caminhão e vendedora.

O grupo de profissionais de saúde foi composto por oito profissionais, dos quais seis são do sexo feminino e dois do sexo masculino, com idades variando entre 29 e 48 anos. Quanto ao nivel de formação profissional sete apresentavam nivel superior, dentre os quais um médico, duas enfermeiras, dois farmacêuticos, uma nutricionista e uma educadora física; o outro profissional apresentava curso profissionalizante de auxiliar de enfermagem.

Os profissionais de saúde apresentavam tempo de formação com variação de sete a 28 anos e experiência de trabalho em serviço público de saúde variando de um a 28 anos. Destaca-se também que três profissionais (um farmacêutico e duas enfermeiras) desempenhavam funções em diferentes níveis de gestão no serviço, dos quais um farmacêutico e duas enfermeiras, o que lhes conferiu maior propriedade sobre a percepção das prioridades e estratégias de intervenção definidos pelo serviço, fundamental para a compreensão da atenção à saúde dispensada às pessoas com diabetes que trabalham. 
A entrevista constitui-se na principal técnica de coleta de dados, utilizada em combinação com a observação participante. Optou-se pela entrevista semiestruturada, que utiliza um roteiro com questões abertas que orientam a temática a ser explorada (12). No grupo de pessoas com diabetes explorou-se a experiência de ser trabalhador com diabetes e a interação com profissionais de saúde. No grupo de profissionais de saúde explorou-se o impacto desta condição crônica no cotidiano do adulto que trabalha e como este aspecto é abordado no âmbito da atenção em saúde.

As entrevistas foram realizadas no CSE no período de março de 2012 a maio de 2013 e transcritas na íntegra. Para a realização da entrevista foi escolhido local reservado, no intuito de não expor os participantes. Antes do início de cada entrevista a pesquisadora apresentou a cada participante os objetivos da pesquisa, o procedimento de entrevista e o Termo de Consentimento Livre e Esclarecido, realizou a leitura do mesmo juntamente com o participante da pesquisa e se dispôs a esclarecer as dúvidas relativas à pesquisa. A duração das entrevistas foi de 30 a 50 minutos.

As pessoas com diabetes foram identificadas com a letra E seguida de número para diferenciá-las (E1, E2, E3... E11) e os profissionais de saúde foram identificados com PS, seguido de número (PS1, PS2, PS3... PS8).

A análise dos dados foi realizada em três etapas conforme preconiza a TFD: codificação aberta, axial e seletiva, que permitiram a identificação das categorias $(10,12)$.

$\mathrm{Na}$ codificação aberta os dados são divididos em incidentes, ou seja, fragmentos de textos que permitem identificar ações, ideias, pensamentos. Estes fragmentos são agrupados de acordo com suas similaridades formando categorias prévias. Prosseguiu-se com a codificação axial, que se constituiu na organização das categorias prévias, neste momento foram identificadas categorias e subcategorias e as relações existentes entre elas. Por fim, seguiu-se com a codificação seletiva, na qual é identificada a categoria central, em torno da qual se articulam as demais categorias e subcategorias explicativas do fenômeno atenção em saúde ao trabalhador com diabetes $(10,12)$.

\section{Resultados}

O processo de análise dos dados permitiu a identificação de quatro categorias: atenção à saúde dividida entre as condições agudas e crônicas; trabalhador com diabetes com acesso limitado ao serviço de saúde; desconhecimento da demanda da população com diabetes mellitus que trabalha e alternativas que aproximam os trabalhadores com diabetes da atenção em saúde.

Estas categorias expressam o conjunto de significados atribuídos pelas pessoas com diabetes e profissionais de saúde à atenção à saúde prestada a pessoa com diabetes que trabalha, as quais se articulam em torno da categoria central: Sempre foi Velado, explicativa do fenômeno em estudo. A seguir, apresenta-se a descrição dos achados em cada categoria. 


\section{Atenção à saúde dividida entre as condições agudas e crônicas}

Os profissionais de saúde ao apresentarem suas percepções sobre a atenção à saúde às pessoas com diabetes mellitus compreendem que o modelo de organização dos serviços de saúde apresenta implicações diretas sobre as ações em saúde prestadas às pessoas com esta condição, conforme revelam as falas a seguir:

Aqui enquanto profissional eu faço orientação que é permitida pela demanda do serviço [...][...] a gente explica, orienta, mas o tempo é curto, não dá pra fazer todas as orientações que você gostaria e, às vezes, quando o paciente chega com alguma dúvida dá pra você esclarecer melhor, mas se ele não tem nenhuma dúvida não dá pra você ficar conversando, por causa da demanda do serviço. (PS8)

Os profissionais de saúde revelam que na rotina dos serviços de saúde as condições agudas e infecciosas convivem com o um aumento crescente das demandas relativas às condições crônicas. A seguir apresenta-se a percepção de um profissional de saúde a respeito da convivência das diferentes demandas no serviço de saúde. "Priorizamos outros programas de saúde, que estavam numa posição mais crítica, como por exemplo, o programa DST/ AIDS. [...] Depois refizemos a nossa programação de metas e o programa de diabetes ele era prioridade um" (PS5).

Frente a esta realidade, os profissionais de saúde percebem-se divididos na atenção a esta diversidade de condições presentes nos serviços de saúde, às quais solicitam ações e estratégias diferenciadas. Esta situação compromete o desenvolvimento de ações adequadas às demandas das condições crônicas, como revela a fala do entrevistado a seguir:

O diabetes hoje na minha concepção eu acho que ele não tem um seguimento na rede de atenção como deveria, se a gente for olhar o prontuário, se realmente a gente seguisse o protocolo, nós não teríamos esta situação em que a gente encontra os pacientes. (PS5)

Embora a necessidade de ações em saúde que atendam às especificidades das condições crônicas seja reconhecida pelos profissionais de saúde e embasada em documentos oficiais e politicas públicas, os profissionais de saúde revelam a necessidade de efetivar estas politicas de saúde na rotina dos serviços, como destacado na fala a seguir:

Então a gente sabe que na verdade a doença crônica no nosso país ainda ela é muito pouco valorizada. (PS3)

O Ministério da Saúde fez todo um movimento inclusive de fazer a vigilância, a notificação das condições crônicas, mas isto não avançou [...] então não notificamos a condição crônica, isto é um atraso em termos de vigilância, porque hoje nós não temos a dimensão do problema. (PS1) 
Como consequência do modelo tradicional em saúde e da convivência das condições agudas e infecciosas, juntamente com as condições crônicas, os profissionais de saúde revelam que na atenção à saúde das pessoas com diabetes as ações são centradas nos sintomas agudos decorrentes de alterações dos niveis de glicemia, que acarretam episódios de hiper ou hipoglicemia. Desta forma, estas ações ocorrem de forma episódica como resposta aos sintomas agudos do diabetes mellitus, como referem estes profissionais:

Eu marco, eu agendo a consulta. Este paciente vem eu foco em cima da queixa apresentada no momento da consulta. (PS3)

A impressão que eu tenho é que a gente olha para o diabetes quando já começa a aparecer a complicação, no momento que a pessoa já está passando mal, não se faz a prevenção. (PS2)

Este tipo de atenção é percebido pelos profissionais como influenciada pelo modelo tradicional de assistência a saúde, o qual é percebido como pouco eficiente na atenção às pessoas com condição crônica, como pode ser observado no relato dos profissionais a seguir:

Os pacientes passavam mensalmente em consulta médica, mas isto não implicava numa boa assistência. Eles procuravam o serviço, passavam com o médico, era o modelo assistencial médico centrado, mas só que de alguma forma não aderiam ao tratamento. (PS3)

$\mathrm{Na}$ percepção das pessoas com diabetes quando a atenção em saúde é baseada no modelo tradicional de saúde, as ações são centradas nos sintomas agudos e, como consequência, compreendidas como uma atenção superficial, por não abarcar a complexidade das demandas decorrentes desta condição, como se pode observar no relato a seguir: "A médica explicou que não pode comer muito aminoácido, comer doce, açúcar. Explicou rapidamente: O senhor tem diabetes deve tomar o remédio diariamente!” (E4).

Além dos sintomas agudos, as pessoas com diabetes revelam o impacto da condição crônica no aspecto emocional e nas atividades cotidianas e ressaltam a importância dos profissionais de saúde estarem atentos a este aspecto:

No início, quando recebi o diagnóstico do diabetes, eu quase enlouqueci por não poder fazer as coisas que eu fazia antes. (E6)

Eu sempre achei que quem tem diabetes deveria fazer uma terapia, porque está muito ligado ao emocional. Eu acho que deveria cuidar do emocional em primeiro lugar. (E2)

Como consequência da atenção centrada nos sintomas agudos da condição crônica e em ações prescritivas pode ocorrer um comprometimento da adesão ao tratamento, conforme expresso na fala deste entrevistado: "Troquei de endocrinologista duas vezes, porque eu não achava que a pessoa me tratava direito, procurei até auxílio em médico particular” (E6). 
Outro aspecto que compromete o seguimento da pessoa com diabetes refere-se à dificuldade de acesso aos serviços de saúde, como será apresentado na categoria a seguir.

\section{Trabalhador com diabetes com acesso limitado ao serviço de saúde}

Na perspectiva dos profissionais de saúde, o horário de funcionamento das unidades de saúde pode limitar o atendimento das pessoas com diabetes que trabalham, pois a maioria dos serviços de saúde atende no horário comercial, como destacado pelos profissionais a seguir:

As unidades ficam abertas só no horário comercial e a maioria trabalha em horário comercial. (PS4)

Uma crítica minha ao serviço, é que ele é feito pra atender as necessidades do prestador de serviço, não pra atender as necessidades de quem necessita do serviço, os usuários. Porque as UBS todas abrem das sete, sete e meia às cinco horas da tarde. Então a população economicamente ativa, que está ficando doente mais cedo, às vezes tem uma doença crônica que não a impede de trabalhar. Mas esta pessoa que trabalha ela vai ter acesso a UBS? Ela tem aí os atestados, mas e os compromissos do trabalho? É mais difícil conseguir oferta de serviço fora do horário comercial. Esta situação é um problema geral. (PS5)

Os profissionais de saúde revelam que as pessoas com condição crônica necessitam realizar o seguimento longitudinal e periódico de sua condição pelos serviços de saúde. Como os serviços de saúde atendem prioritariamente em horário comercial, com exceção dos serviços de pronto atendimento, os trabalhadores com diabetes podem encontrar dificuldade em realizar o seguimento de sua condição crônica, pois necessitará solicitar afastamentos periódicos do trabalho.

Para estes profissionais a limitação nos horários de atendimento das unidades básicas e serviços especializados de atenção em diabetes pode resultar na busca pelos serviços de pronto-atendimento. Como consequência, não haverá um seguimento longitudinal das pessoas com diabetes, o que pode comprometer a adesão destas pessoas às orientações e tratamentos prescritos, bem como ações preventivas e de promoção em saúde, podendo resultar em maior comprometimento da pessoa com condição crônica e na sobrecarga de serviços de pronto atendimento. Este aspecto pode ser observado na percepção do profissional, destaca a seguir, sobre a percepção da elevada demanda de pessoas com diabetes nos serviços de pronto atendimento. "Será que é porque são pessoas que encontraram as portas fechadas nas unidades básicas e estão agora procurando o pronto atendimento? Ou será que é pós trabalho?” (PS1).

Na perspectiva da pessoa com diabetes, o acesso limitado ao serviço de saúde é percebido pela dificuldade de agendamento de consultas e exames. 
A fala a seguir revela esta situação. "É até difícil marcar controle, exame de sangue. A gente acaba não sentindo nada e acaba deixando pra lá, porque precisa trabalhar" (E1).

Desta forma, na perspectiva de profissionais de saúde e pessoas com diabetes o sistema de saúde não favorece o seguimento da pessoa com diabetes que trabalha e como consequência observa-se o desconhecimento da demanda da população com diabetes mellitus que trabalha, apresentado na categoria a seguir.

\section{Desconhecimento da demanda da população com diabetes mellitus que trabalha}

Os profissionais de saúde identificam que o DM é uma condição altamente prevalente e está presente nos diferentes níveis de atenção em saúde, principalmente no pronto-atendimento. Este aspecto, somado a não utilização dos protocolos, que atuam como guias na investigação clínica e na definição das condutas a serem realizadas pelos profissionais de saúde, dificultam o conhecimento da demanda das pessoas com diabetes e do quanto esta condição afeta a população de um modo geral. Este desconhecimento é apresentado na fala do profissional a seguir:

Aqui mesmo a gente começou a fazer novamente o levantamento e eu tenho a impressão que nós temos um número bastante expressivo de pacientes diabéticos, mas eu desconheço a situação dos diabéticos, por que esta condição precisa ser monitorada. (PS2)

Para os profissionais de saúde o desconhecimento da demanda e o seguimento pontual desta condição crônica dificulta o desenvolvimento de uma intervenção humanizada, que possibilite compreender o sujeito em sua integralidade, de forma a abarcar os aspectos históricos, sociais, políticos e culturais, articulado com meio ambiente e sociedade no qual o adoecido se insere. A fala a seguir revela a percepção do profissional de saúde sobre a atenção prestada à pessoa com DM que trabalha:

Costuma ser geral, quando o paciente questiona a gente direciona a orientação para aquele questionamento que ele tá fazendo, geralmente quando é alguém que vê alguma dificuldade, vê algum impedimento ele vai questionar ai ele vai ser orientado. (PS5)

Assim, os profissionais de saúde revelam que as ações em saúde são gerais e são especificadas somente quando a pessoa com diabetes apresenta ao profissional a sua demanda. Desta forma, embora muitas pessoas com DM sejam diagnosticadas ainda em idade produtiva, as implicações do DM no trabalho não se constituem em um aspecto abordado pelo profissional na rotina do serviço de saúde, como revelado pelo profissional na fala a abaixo. 
A gente não pergunta especificamente sobre o trabalho. (PS4)

Estes pacientes, grande parte estava em idade ainda produtiva, eles ainda estavam no mercado de trabalho, com muitas limitações por causa do diabetes, tinha paciente, por exemplo, que quando chegavam não tinha jeito, tinha que entrar com insulina, eles até choravam e questionavam: “Tenho que trabalhar como é que vou fazer?". (PS2)

Soma-se a este aspecto, o fato de que os profissionais de saúde não dispõem de protocolos ou orientações definidas em documentos nacionais, como portarias ou políticas públicas, para nortear a intervenção junto à pessoa com diabetes que trabalha. Deste modo, fica a critério dos profissionais de saúde elencar as informações necessárias para orientar as ações em saúde, como apresenta o relato destes profissionais:

Eu gosto de ver sempre o contexto onde a pessoa está inserida, se ela trabalha, como é no serviço, se tem onde guardar a insulina, se tem como levar uma fruta ou alguma coisa para comer no meio do serviço pra não ficar oito horas sem se alimentar, quem que pode ajudar no caso de passar mal, que orientação que a gente pode passar para esta pessoa. (PS5)

Eu preciso saber os horários que ela entra no trabalho, que horário que ela tem um intervalo, se no trabalho tem um horário pra ela fazer essa refeição, como é que é essa dinâmica no trabalho dela. Sem conhecer isso eu vou tá passando uma orientação que não cabe na rotina dela. (PS6)

A preocupação destes profissionais de saúde em compreender as implicações da condição crônica no cotidiano dos adoecidos aproxima-se de uma importante percepção das pessoas com DM, pois de acordo com estas pessoas a condição crônica solicita uma intervenção em saúde que seja capaz de abranger o impacto social e emocional desta condição, como revelado a seguir:

Aí você pensa o pior né, que você vai morrer! [...] Então no início foi um "baque" tanto é que eu emagreci quase 20 quilos, porque no caso até se deprime, no meu caso até aceitar isso foi uns dois anos. (E6)

Para as pessoas com DM esta condição pode acarretar implicações no trabalho, podendo comprometer seu rendimento, bem como a adesão às orientações realizadas pelos profissionais de saúde:

Pessoa que tem diabetes não aguenta muito, não aguenta mesmo. Eu não aguento o pique que eu aguentava antes de ter diabetes. (E8)

No trabalho não dá pra fica comento toda hora, eu acho que trabalho é trabalho [...] você perde muito tempo nesse negócio de comer devagar. (E4) 
Desta forma, as demandas relativas às implicações da condição crônica no cotidiano, destacando-se a atividade de trabalho, não se constitui em um aspecto a ser avaliado pelo profissional de saúde na rotina do serviço. A realização de avaliações e orientações relacionadas ao trabalho fica a critério do profissional, bem como do adoecido ao apresentar as dificuldades encontradas no cotidiano de trabalho como queixa. Como consequência, pouco se conhece sobre as implicações do DM para o trabalho e de como a rotina de trabalho pode dificultar a adesão às ações de autocuidado.

Frente à percepção de profissionais de saúde e pessoas com DM sobre o desconhecimento das implicações desta condição no trabalho os entrevistados apresentam suas percepções sobre as alternativas que aproximam os trabalhadores com diabetes da atenção em.

\section{Alternativas que podem aproximar os trabalhadores com diabetes da atenção em saúde}

Os profissionais de saúde, ao identificarem as limitações de acesso dos trabalhadores com diabetes aos serviços de saúde, resgataram experiências realizadas em outros serviços de saúde compreendendo-as como favorecedoras do acesso desta população, como pode ser observado nas falas a seguir:

Já existem algumas unidades básicas de saúde com horários ampliados até às dezenove horas, às vezes ate às vinte e uma horas, mas não são todas, não são todos os bairros, não são todos os locais, aí eles acabam frente às necessidades procurando os serviços de pronto atendimento que é vinte e quatro horas. (PS5)

Aqui na unidade a gente tentou fazer algumas coisas à noite, mas a gente não conseguiu, mas há outras cidades, que uma vez por semana começam a trabalhar mais tarde e ficam até parte da noite atendendo, até oito horas, nove horas, para atender a população que trabalha. (PS4)

Na perspectiva das pessoas com diabetes, os grupos de educação em diabetes são compreendidos como espaços importantes para desenvolver formas de enfrentamento da condição crônica e compartilhar dúvidas, angústias, estratégias desenvolvidas no cotidiano, além de oferecer informações sobre os aspectos biológicos da doença, como destacado pelo entrevistado:

A partir do momento que eu comecei a frequentar o grupo as coisas foram sendo mais esclarecidas, o porquê, de como acontece no organismo. [...] Porque ao ser diagnosticado com diabetes você pensa o pior, que você vai morrer, que você terá que tomar insulina para o resto da vida. Mas com o decorrer do tempo eu fui procurando me informar, para poder estar realmente apto para essa nova situação. (E6) 
As pessoas com diabetes revelam a necessidade de um cuidado em saúde que abarque não somente os aspectos biológicos, mas que possibilitem acolher o impacto desta condição em seu cotidiano, no qual se destacaram as atividades de trabalho. Na perspectiva dos profissionais de saúde, os aspectos relativos às implicações entre diabetes e trabalho são pouco explorados e conhecidos na rotina de serviços de saúde, sendo este conteúdo apresentado nas diferentes categorias e expresso a seguir na categoria central.

\section{Categoria Central: Sempre foi velado}

A atenção à saúde da pessoa com diabetes que trabalha é compreendida pelos profissionais de saúde e pessoas com diabetes como centrada nos aspectos clínicos, relacionados aos sintomas agudos decorrentes desta condição. As percepções e ações de ambos constroem-se em torno deste aspecto, assim para o profissional de saúde o modelo de atenção vigente e a elevada demanda nos serviços de saúde não permitem abarcar aspectos relacionados ao impacto desta condição em outros contextos da vida como o trabalho, o lazer, as relações pessoais, dentre outros aspectos.

A pessoa com diabetes, por sua vez, não reconhece o serviço de saúde como um contexto de apoio às suas demandas sociais e emocionais e, como consequência, não apresenta a demanda relacionada às dificuldades em conciliar as ações de autocuidado com as condições de trabalho, mantendo as implicações de sua condição crônica no trabalho veladas nas interações que estabelece com os profissionais de saúde. As falas a seguir são representativas deste fenômeno:

Saber que você é doente. [...] No início não foi fácil não, principalmente neste aspecto do trabalho, você ter que estar disposto para trabalhar. Alguns patrões que eu tive se ressentiram disso, mas nunca foi falado, violado... sempre foi velado. (E6)

Não sei se é um sentimento de vergonha, mas eu acho que as pessoas inicialmente elas acabam não revelando, convivendo no trabalho, às vezes até para os familiares elas acabam deixando sem con$\operatorname{tar}[\ldots]$ (PS6)

As necessidades do trabalhador com diabetes são percebidas como veladas também no âmbito das políticas públicas e diretrizes em saúde, pela inexistência de dispositivos legais que garantam ao trabalhador com DM as condições adequadas para a realização do autocuidado no trabalho e, consequentemente, para a manutenção de sua produtividade. Para os profissionais de saúde, sem a existência destes dispositivos, não há respaldo para intervir frente a esta problemática. 


\section{Discussão}

O significado da atenção à saúde da pessoa com diabetes que trabalha foi construído a partir da perspectiva dos adoecidos e profissionais de saúde. Embora partam de perspectivas diferentes, ambos significaram como sempre foi velado na atenção à saúde os aspectos relacionados ao trabalho, bem como a outras demandas emocionais, sociais e econômicas.

De acordo com o Interacionismo Simbólico, os significados são construídos e partilhados nas interações sociais e influenciam as ações e comportamentos (13).

Este referencial teórico permite compreender que na interação entre profissionais de saúde e pessoas com diabetes são partilhados os significados atribuídos à atenção em saúde e à condição crônica, os quais permeiam as interações e relações estabelecidas e delineiam as ações que ocorrem nesta interação. Assim, embora ambos partilhem de que é necessária uma atenção em saúde que abarque aspectos emocionais e as implicações da condição crônica nos diferentes contextos de vida, o desenvolvimento destas ações esbarra no modelo de atenção vigente e na elevada demanda nos serviços de saúde. Estes aspectos não permitem ao profissional de saúde ampliar a atenção, ficando a cargo da pessoa com diabetes apresentar as demandas relacionadas ao trabalho na interação que estabelece com o profissional.

No Brasil, as ações em saúde são realizadas com base no modelo tradicional de atenção à saúde, que se caracteriza pela prestação de cuidados aos eventos agudos e àqueles decorrentes da agudização das condições crônicas, de forma fragmentada, episódica e reativa. A assistência é centrada no ato prescritivo, com a valorização da dimensão biológica e não considera outros determinantes do processo de saúde-doença, como os aspectos sociais, econômicos e culturais $(14,15)$. Este modelo de atenção à saúde é projetado para fornecer acesso rápido ao serviço, com consultas breves, centradas na exclusão de diagnósticos alternativos mais graves, dependêntes de exames laboratoriais, nas prescrições e tratamento de sintomas e sinais (14-18).

A persistencia deste modelo de atenção tradiciondal no sistema de saúde decorre da convivencia entre as demandas das condições crônicas, juntamente com uma elevada demanda de condições agudas e infecciosas. Frente a esta realidade, os programas e ações em saúde são direcionados às condições agudas, não enfatizando ou criando condições adequadas para o seguimento da condição crônica. Isto ocorre, pois no Brasil há uma situação de tripla carga de doenças, na qual há aumento das condições crônicas, em decorrência do envelhecimento da população e dos hábitos alimentares adotados, concomitantemente com a persistência das doenças infecciosas e das doenças agudas. Desta forma, os sistemas de saúde permanecem organizados para atender às condições agudas com base no modelo tradicional de cuidados de saúde, o que impossibilita a apresentação de um plano de gerenciamento adequado às especificidades e exigências das condições crônicas (15).

Frente a este modelo de atenção em saúde, as pessoas com diabetes compreendem as ações em saúde como superficial e, como consequência, identificam que há uma fragilização da interação e vinculo estabelecido com o profissional de saúde, aspecto fundamental para a adesão às orientações 
realizadas. Soma-se a isto, o fato de que este modelo de atenção à saúde, não possibilita que as dificuldades encontradas para o desempenho das atividades cotidianas, decorrentes da condição crônica, sejam abordadas na atenção à saúde. Como consequência, as pessoas com diabetes podem permanecer com dúvidas, angustias e, consequentemente, com o desempenho prejudicado em atividades cotidianas como o trabalho.

Este aspecto pode ser compreendido a luz do IS, o qual apresenta que na gestão cotidiana da condição crônica há um impacto recíproco entre trajetória da doença e gestão da vida cotidiana, na qual as mudanças nas condições de doença não afetam somente a trajetória de doença, mas afetam também a gestão da vida cotidiana, assim como o contexto estrutural, no qual a gestão ocorre, tem implicações importantes para a gestão da doença (19).

A análise da atenção prestada à pessoa com condição crônica permite compreender que não há uma escuta e valorização, pelos profissionais de saúde, da experiência e conhecimento da pessoa com diabetes sobre a sua condição crônica. Este aspecto decorre do modelo de atenção tradicional no qual o conhecimento é compreendido como centrado no profissional de saúde, tendo como pressuposto que a informação disponibilizada constitui-se em condição suficiente para alterar comportamentos, tendo por objetivo a adesão às recomendações do profissional (20). Neste modelo, usuários do sistema de saúde são remetidos a um papel passivo, de receptor de cuidados médicos (16).

As pessoas com diabetes revelam que a atenção centrada nos aspectos prescritivos e biológicos não abrange demandas relacionadas aos aspectos sociais e emocionais, consideradas por elas como fundamentais no processo de aceitação e desenvolvimento de formas de enfrentamentos frente às dificuldades impostas pelo diabetes. Estes achados corroboram com achados prévios $(21,22)$ que identificam que a vivência da condição crônica afeta muito mais que a esfera biológica, pois interfere de diferentes formas nos estilos de vida das pessoas acometidas, da família e de seu grupo social.

As doenças tomadas como objetos concretos, esvaziadas de significado social, cultural ou psíquico, fazem com que frequentemente o médico e os profissionais de saúde as vejam de modo diverso das preocupações dos adoecidos. Porém, assim como as pessoas com diabetes, os profissionais veem o mundo com base em elementos ideativos, simbólicos, que permeiam a sociedade e o tempo em que vivem, de modo que sua prática possa adotar condutas próprias nas quais as evidências teóricas são filtradas, simultaneamente pela experiência e representações muito embora, sempre protegidos pelo escudo da racionalidade científica (23).

Assim, embora profissionais de saúde e pessoas com diabetes partam de perspectivas diversas, ambos compreendem a importância de uma abordagem integral, porém esta abordagem esbarra na restrição dos horários de atendimento dos serviços de saúde que, em geral, coincidem com o horário de trabalho dos adoecidos, o que acarreta como consequência a busca pelos serviços de pronto atendimento, comprometendo o seguimento longitudinal da pessoa com DM. 
A pessoa com diabetes em decorrência de sua condição crônica necessita de seguimento periódico e longitudinal pelo serviço com base em ações planejadas e com enfoque na prevenção de agravos e complicações (24). Esta atenção direcionada à pessoa com condição crônica deve envolver avaliações sistemáticas e individualização do cuidado, considerando as necessidades e valores da pessoa $(17,18)$.

A análise das percepções de profissionais de saúde revela que embora identifiquem que há muitas pessoas com DM em idade produtiva, as implicações desta condição crônica para o trabalho não se constituem em um aspecto abordado pelo profissional na rotina do serviço de saúde. Estes profissionais alegam que não dispõem de protocolos, orientações ou politicas públicas para a abordagem deste aspecto, desta forma fica a critério dos profissionais de saúde a sua abordagem, o que resulta no desconhecimento da demanda da pessoa com diabetes que trabalha.

Para melhorar a atenção à saúde das pessoas com condição crônica é preciso rever o modelo de atenção em saúde, superando a atenção à saúde fragmentada, para um sistema proativo, integrado e continuo que seja focado na pessoa e na família e voltado para a prevenção de doenças e complicações e para a promoção da saúde $(17,20)$, visando uma atenção articulada e integral à pessoa com diabetes $(14,15)$.

A ausência de instrumentos legais que garantam as necessidades da pessoa com diabetes no contexto de trabalho pode ser compreendida como uma forma de manter esta questão velada também no âmbito das políticas públicas sociais e de saúde. Desta forma, revelar as implicações recíprocas entre diabetes e trabalho perpassa pela necessidade de capacitar os profissionais de saúde para avaliarem estes aspectos e realizarem as orientações necessárias e pela criação de leis que garantam o direito da pessoa com diabetes de realizar as modificações necessárias no contexto do trabalho para viabilizar as ações de autocuidado, bem como manter sua inserção e permanência no trabalho.

\section{Considerações finais}

Os significados atribuídos à atenção a saúde da pessoa com diabetes que trabalha permitem concluir que as implicações da condição crônica no cotidiano, enfatizando-se as atividades de trabalho, são pouco exploradas pelos profissionais de saúde e pessoas com diabetes. Este estudo permite compreender que é preciso superar o modelo tradicional de atenção à saúde e buscar estratégias em saúde que permitam compreender a pessoa com condição crônica em sua integralidade, partindo-se das experiências e conhecimentos adquiridos por elas na orientação e educação em saúde.

Os profissionais de saúde ao ampliarem o olhar para uma atenção em saúde humanizada e integral podem redefinir os significados atribuídos à experiência do diabetes e, a partir disto, reorientar a ação desenvolvida junto às pessoas com diabetes que trabalham. Nesta interação as pessoas com diabetes podem redefinir os significados atribuídos ao DM, bem como a percepção de si enquanto trabalhador com uma condição crônica, o que pode colaborar com a adesão às ações relacionadas ao autocuidado no contexto do trabalho. 


\section{Financiamento}

Conselho Nacional de Desenvolvimento Científico e Tecnológico (CNPq) por meio de concessão de bolsa de estudo (Processo $n^{\circ}$ 157836/2013-4).

\section{Conflitos de interesse}

Os autores declaram que não há conflito de interesse.

\section{Referencias}

1. Shaw JE, Sicree RA, Zimmet PZ. Global estimates of the prevalence of diabetes for 2010 and 2030. Diabetes Res Clin Pract. 2010; 87:4-14.

2. World Health Organization. World health statistics [internet]. Geneva: World Health Organization; 2011. [citado 12 jan 2015]. Disponible en: http://www.who.int/whosis/whostat/EN_WHS2011_Full.pdf

3. Tunceli K, Bradley CJ, Nerenz D, Willians LK, Pladevall M, Lafata JE. The impact of diabetes on employment and work productivity. Diabetes Care. 2005;28(11):2662-7.

4. Herquelot E, Gueguen A, Bonenfant S. Impact of diabetes on work cessation: data from the Gazel cohort study. Diabetes Care [internet]. 2011 [citado 12 jan 2015];34(6):1344-49. Disponivel em: http://care. diabetesjournals.org/content/34/6/1344.full

5. Detaille S, Haafkens JA, Hoekstra JB, Dijk FJH. What employees with diabetes mellitus need to cope at work: views of employees and health professionals. Patient Educ Couns [internet]. 2006;64(3):183-90. Disponível em: http://www.pec-journal.com/article/ S0738- 3991(06)00005-X/pdf

6. González GR, Castro MG, Pérez RS, Alonso ED. Problemática laboral em um grupo de personas com diabetes mellitus. Rev Cubana Endocrinol [internet]. 2009;20(6):89-103. Disponivel em: http://scielo. sld.cu/scielo.php?script=sci_arttext\&pid=S1561- 29532009000300003

7. Thomas EA. Diabetes at work: A grounded-theory pilot study. AAOHN J. 2011;59(5):213-20.

8. Fukunaga LL, Uehara DL, Tom T. Perceptions of diabetes, barriers to disease management, and service needs: a focus group study of working adults with diabetes in Hawaii. Prev Chronic Dis [internet]. 2011;8(2):1-8. Disponivel em: http://www.cdc.gov/pcd/issues/2011/ mar/09_0233.htm

9. American Diabetes Association. Diabetes and employment. Diabetes Care. 2009;37(1):S80-4.

10. Charmaz KA. Construção da teoria fundamentada nos dados: guia prático para as abordagens qualitativas. Porto Alegre: Artmed; 2009.

11. Carvalho VD, Borges LO, Rêgo DP. Interacionismo simbólico: origens, pressupostos e contribuições aos estudos em psicologia social. Psicol Ciênc Prof. 2010;30(1):146-61.

12. Strauss A, Corbin J. Pesquisa qualitativa: técnica e procedimentos para o desenvolvimento da teoria fundamentada. 2a ed. Porto Alegre: Artmed; 2008. 
13. Blumer H. Symbolic interactionism: perspective and method. Berkeley: University of California; 1969.

14. Maltha DC, Merhy EE. O percurso da linha do cuidado sob a perspectiva das doenças crônicas não transmissiveis. Interface. 2010;14(34):593-605.

15. Mendes EV. O cuidado das condições crônicas na atenção primária à saúde: o imperativo da consolidação da estratégia da saúde da família [internet]. Brasília: Organização Pan-Americana da Saúde; 2012 [citado 10 jan 2015]. Disponivel em: http://bvsms.saude.gov.br/bvs/ publicacoes/cuidado_condicoes_atencao_primaria_saude.pdf

16. Almeida LM. Os serviços de saúde pública e o sistema de saúde. Rev Port Sau Pub [internet]. 2010;28(1):79-92. Disponível em: http://www. scielo.oces.mctes.pt/pdf/rpsp/v28n1/v28n1a09

17. Wagner EH. Chronic disease management: what will it take to improve care for chronic illness? Eff Clin Pract. 1998;1(2): 2-4.

18. Wagner EH, Austin BT, Davis C, Hindmarsh M, Schaefer J, Bonomi A. Improving chronic illness care: translating evidence into action. Health Affairs. 2001;20(6):64-78.

19. Corbin J, Strauss A. A grounded theory research: procedures, canons and evaluative criteria. Qual Sociol. 1990;13(1):3-21.

20. Bodenheimer T, Wagner EH, Grumbach K. Improving primary care for patients with chronic illness: the chronic care model, part 2. JAMA [internet]. 2002 [citado 2013 jul 10];288(15):1909-14. Disponível em: http://www.uft-a.com/PDF/JAMABodenheimer10-02.pdf

21. Faria APS, Bellato RA. Compreensão do fenômeno condição crônica por diabetes mellitus a partir da experiência de adoecimento. Rev Eletr Enf [internet]. 2010;12(3):520-7. Disponivel em: http://www.fen. ufg.br/fen_revista/v12/n3/v12n3a15.htm

22. Martins LM, França APD, Kimura M. Qualidade de vida de pessoas com doença crônica. Rev Latino-Am Enferm [internet]. 1996 [citado 2014 mai 2];4(3):35-18. Disponivel em: http://www.scielo.br/pdf/rlae/ $\mathrm{v} 4 \mathrm{n} 3 / \mathrm{v} 4 \mathrm{n} 3 \mathrm{aO} 2$

23. Barsaglini RA. As representações sociais e a experiência com o diabetes: um enfoque socioantropológico. Rio de Janeiro: FIOCRUZ; 2011.

24. Organização Mundial de Saúde. Cuidados inovadores para condições crônicas: componentes estruturais de ação: relatório mundial [internet]. Brasília: OMS; 2003. Disponível em: www.who.int/chp/knowledge/publications/icccportuguese.pdf 